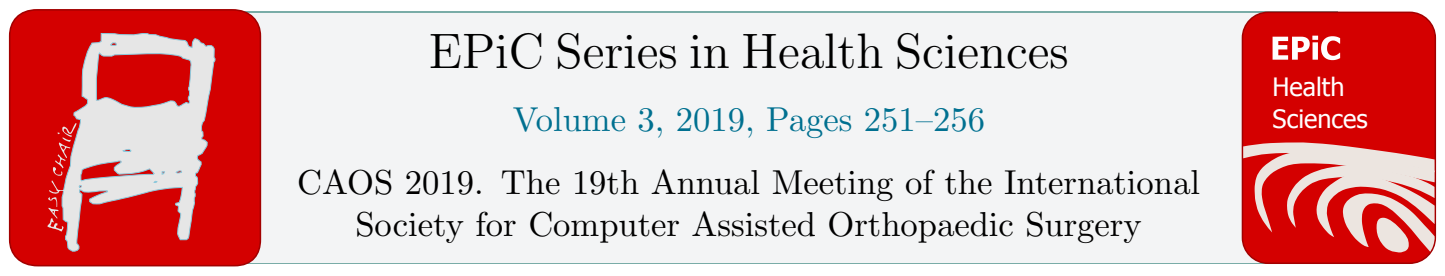

\title{
Trends in Cup Position Utilizing Computer- Assisted Navigation During Total Hip Arthroplasty
}

\author{
Rachel R. Mays ${ }^{1}$, Jessica R. Benson ${ }^{1}$, Jeffrey M. Muir ${ }^{1}$, \\ Peter B. White ${ }^{2}$ and Morteza Meftah ${ }^{3}$ \\ ${ }^{1}$ Department of Clinical Research, Intellijoint Surgical, Waterloo, Ontario, Canada \\ 2Lake Erie College of Osteopathic Medicine, Erie, Pennsylvania, USA \\ ${ }^{3}$ Division of Adult Reconstructive Surgery, NYU Langone Orthopedic Hospital, NYU \\ Langone Health, New York, New York, USA \\ r.mays@intellijointsurgical.com, morteza.meftah@nyulangone.org
}

\begin{abstract}
Proper positioning of the acetabular cup deters dislocation after total hip arthroplasty (THA). The concept of a safe zone (SZ) for acetabular component placement was first characterized by Lewinnek et al. and furthered by Callanan $e t$ al. The SZ concept remains widely utilized and accepted in contemporary THA practice; however, components positioned in this SZ still dislocate. This study sought to characterize current mass trends in cup position identified across a large study sample of THA procedures completed by multiple surgeons. This retrospective, observational study reviewed acetabular cup position in 1,236 patients who underwent THA using computer-assisted navigation between July 2015 and November 2017. The overall mean cup position of all recorded cases was $21.8^{\circ}\left( \pm 7.7^{\circ}, 95 \% \mathrm{CI}=6.7^{\circ}, 36.9^{\circ}\right)$ of anteversion and $40.9^{\circ}\left( \pm 6.5^{\circ}, 95 \% \mathrm{CI}=\right.$ $\left.28.1^{\circ}, 53.7^{\circ}\right)$ of inclination. For both anteversion and inclination, $65.5 \%(809 / 1236)$ of acetabular cup components were within the Lewinnek SZ and 58.4\% (722/1236) were within the Callanan SZ. Acetabular cups were placed a mean of $6.8^{\circ}$ of anteversion (posterior/lateral approach: $7.0^{\circ}$, anterior approach: $5.6^{\circ}$ ) higher than the Lewinnek and Callanan SZs whereas inclination was positioned $0.9^{\circ}$ higher than the reported Lewinnek SZ and $3.4^{\circ}$ higher than the Callanan SZ. Our data shows that while the majority of acetabular cups were placed within the traditional SZs, the mean anteversion orientation is considerably higher than those suggested by the Lewinnek and Callanan SZs. The implications of this observation warrant further investigation.
\end{abstract}




\section{Introduction}

Total hip arthroplasty (THA) is one of the most successful procedures in orthopedics today and is associated with reproducible, positive clinical outcomes (Charnley, 1972). The incorrect positioning of acetabular components during THA also increases the risk for a multitude of other post-surgical complications, including accelerated component wear, joint instability, impingement and reduced range of motion (Elkins, Callaghan, \& Brown, 2015). Preventing dislocation through optimization of cup position was first introduced to the literature (Lewinnek, Lewis, Tarr, Compere, \& Zimmerman, 1978), who observed a greater dislocation rate for acetabular cup components positioned outside a delegated safe range of version and inclination values. Recent studies (Abdel, von Roth, Jennings, Hanssen, \& Pagnano, 2016; Reina et al., 2017); however, have questioned the validity of the concept of the SZ itself and whether the SZ needs to be adjusted according to each patient. Furthermore, there is a distinct lack evidence to indicate that surgeons consistently place components within these defined ranges (McCollum \& Gray, 1990).

Utilizing an imageless, 3-dimensional computer-assisted navigation system, the goal of this large retrospective, observational study was to characterize contemporary mass trends in acetabular cup position (specifically anteversion and inclination) identified across a large study sample. The secondary goal was to determine if the surgical approach (posterior/lateral or anterior) affected the rate in which these components were placed in the SZs.

\section{Methods}

This retrospective, observational study reviewed acetabular cup position for patients who underwent THA using a computer-assisted navigation device. Patients were eligible for inclusion in the study if they underwent a THA procedure performed via the posterior, lateral or direct anterior approach, between February 2016 and November 2017 at one of fourteen institutions in which the navigation tool was utilized. This study utilized Intellijoint HIP® (Intellijoint Surgical, Inc., Waterloo, Ontario), an imageless, 3D mini-navigation tool that provides quantitative, real-time measurements for cup position and change in leg length and offset during THA. Statistical comparisons were made with alpha set a priori at 0.05 . Outcomes are presented as mean (standard deviation [95\% confidence interval]). Continuous variable means were compared using the Student's t-tests. Categorical variable means were compared using chi-square tests.

\section{Results}

A total of 1,236 cases were performed during the study period and were included in the analysis. The mean cup position of all recorded cases was $21.8^{\circ}\left( \pm 7.7^{\circ}, 95 \% \mathrm{CI}\left[6.7^{\circ}, 36.9^{\circ}\right]\right)$ of anteversion and $40.9^{\circ}\left( \pm 6.5^{\circ}, 95 \% \mathrm{CI}\left[28.1^{\circ}, 53.7^{\circ}\right]\right)$ of inclination. When stratified by surgical approach (anterior or posterior/lateral), the mean cup position for anterior approach cases was $20.6^{\circ}\left( \pm 6.4^{\circ}, 95 \% \mathrm{CI}\left[8.1^{\circ}\right.\right.$, $\left.\left.33.1^{\circ}\right]\right)$ of anteversion and $37.8^{\circ}\left( \pm 5.4^{\circ}, 95 \%\right.$ CI $\left.\left[27.2^{\circ}, 48.4^{\circ}\right]\right)$ of inclination. The mean cup position in cases using the posterior/lateral approach was significantly higher than the anterior approach for both anteversion $\left(22.0^{\circ} \pm 7.9^{\circ}, 95 \%\right.$ CI $\left.\left[6.5^{\circ}, 37.5^{\circ}\right], \mathrm{p}<0.001\right)$ and inclination $\left(41.5^{\circ} \pm 6.6^{\circ}, 95 \%\right.$ CI $\left[28.6^{\circ}\right.$, $\left.\left.54.3^{\circ}\right], \mathrm{p}<0.001\right)$.

Across all recorded cases, $90 \%$ of anteversion measurements and $95 \%$ of inclination measurements fell within $\pm 10^{\circ}$ of the mean. For the anterior approach, the $\pm 10^{\circ}$ range incorporated $89.7 \%$ of anteversion and $93.3 \%$ of inclination values, while for the posterior/lateral approach, $91.3 \%$ of 
anteversion and $96 \%$ of inclination values were within $\pm 10^{\circ}$ of the mean (Table 1). Across all cases, the range of anteversion measurements that incorporated $95 \%$ of cup orientations was $\pm 12.5^{\circ}$. For inclination, a range of $\pm 10^{\circ}$ was required to incorporate $95 \%$ of measurements. Similar results were reported for anterior approach, where ranges of $\pm 13.5^{\circ}$ and $\pm 10.5^{\circ}$ were required to incorporate $95 \%$ of anteversion and inclination measurements, respectively. Finally, for the posterior/lateral approach, range of $\pm 12.5^{\circ}$ and $\pm 9^{\circ}$ incorporated $95 \%$ of cups for anteversion and inclination measurements, respectively.

A total of $65.5 \%(809 / 1236)$ of all included cases were inside the Lewinnek SZ for both anteversion and inclination, while 58.4\% (722/1236) of all cases were inside the Callanan SZ ( $<<0.001)$. More specifically, for inclination, $95.3 \%$ (1025/1236) of cup components were placed in the Lewinnek SZ and $82.9 \%(1178 / 1236)$ were placed within the Callanan SZ, while only $67.4 \%(833 / 1236)$ of cups were placed in the common SZs for anteversion. Significantly more acetabular components were placed within the accepted SZs for inclination than for anteversion $(\mathrm{p}<0.001$ for both Lewinnek and Callanan SZs) (Figures 1,2).

\section{Discussion}

The proper positioning of the acetabular component in THA is imperative in establishing joint stability and longevity, as optimum cup positioning can reduce the risk of negative outcomes such as accelerated wear, impingement and, most importantly, dislocation (Elkins et al., 2015). The Lewinnek and Callanan SZs were intended to avoid the placement of acetabular cup components in orientations associated with a greater risk of dislocation by providing suggested ranges for inclination and anteversion in which the rate of dislocation or instability was thought to be lower. These SZs are widely accepted and utilized in contemporary clinical practice; however, current studies suggest they are potentially ineffective, as many hips within these SZs nevertheless dislocate (Abdel et al., 2016). The purpose of this study was to observe and compare trends in acetabular cup placement across a large study sample, in order to identify current trends in acetabular component placement and whether this correlates to these previously designated zones.

Our data shows that while the majority of acetabular components are placed in the Lewinnek and Callanan designated SZs, acetabular cup components were placed, on average, with anteversion $7^{\circ}$ higher than the suggested mean of the SZ, whereas inclination was more closely mirrored the suggested SZ and were placed within $1^{\circ}$ of the designated SZs. The evidence in the literature and surgeon consensus suggest that these target values may not be as useful as once thought, as they do not always forecast dislocation rates (Lewinnek et al., 1978). It is likely that the preferred cup position lies outside of these safety zones in some cases and more advanced analysis is necessary to determine the correct, patient-specific target. With a better understanding of the factors affecting optimal cup positioning, a customized approach to acetabular orientation may prove to be most effective at minimizing impingement, dislocations and wear. 


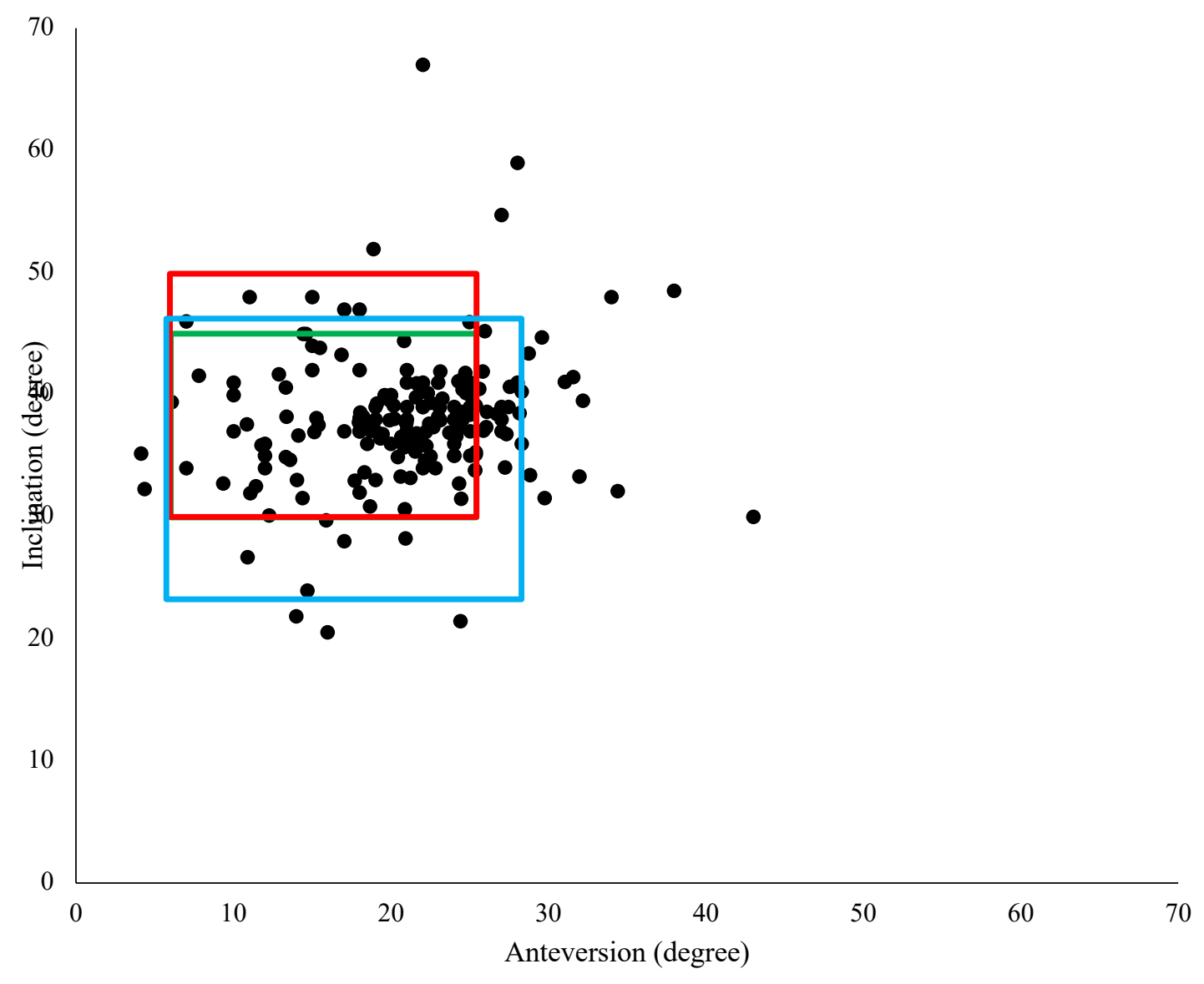

Figure 1. Scatter plot of all the cup position for anterior approach THA. The Lewinnek SZ is denoted in the red box, Callanan SZ in the green box, the $95 \%$ confidence interval of the mean is in the blue box, and the mean and standard deviation are in the blue dash box. 


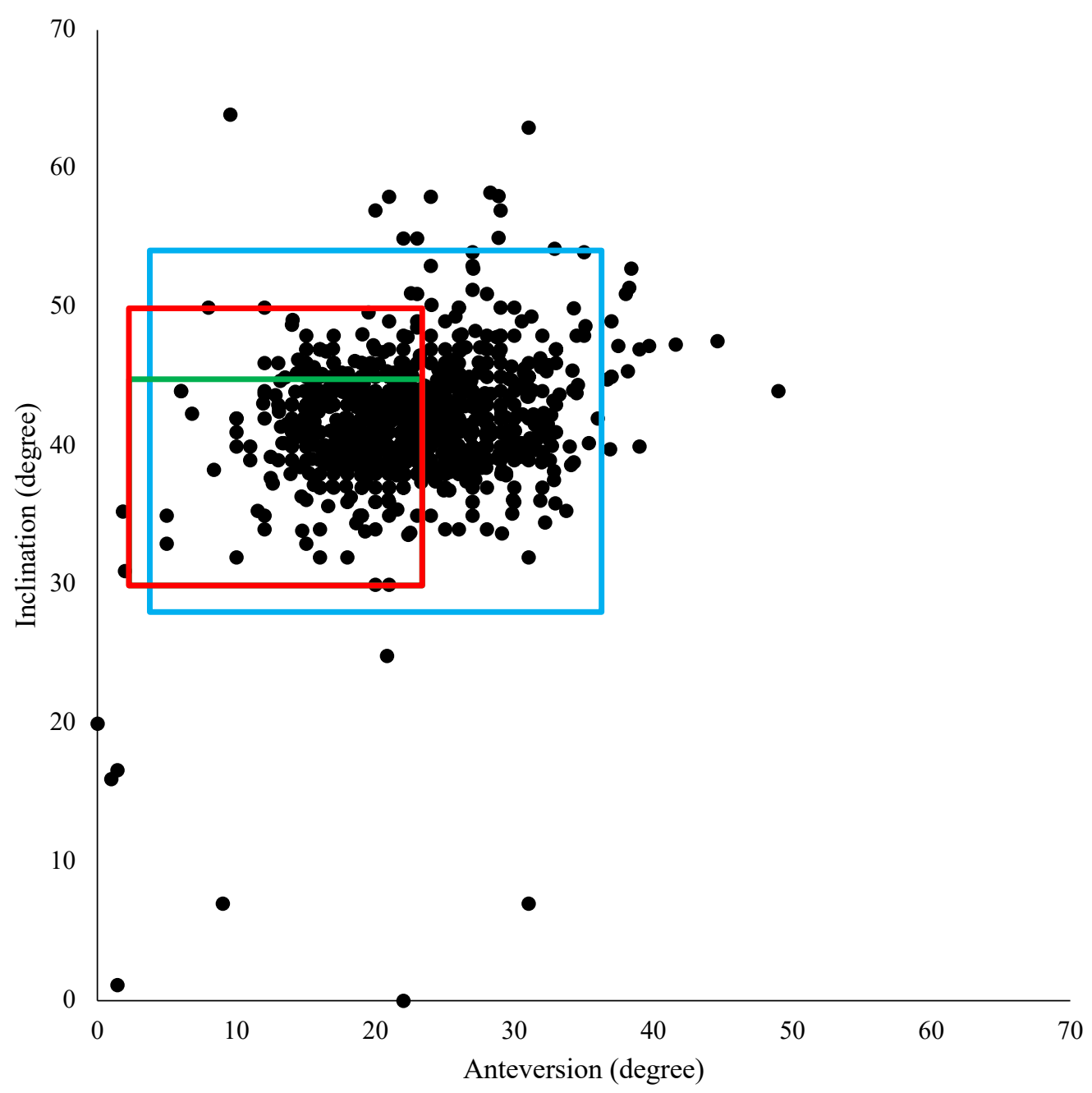

Figure 2. Scatter plot of all the cup position for posterior/lateral approach THA. The Lewinnek SZ is denoted in the red box, Callanan SZ in the green box, the $95 \%$ confidence interval of the mean is in the blue box, and the mean and standard deviation are in the blue dash box. 


\section{References}

Abdel, M. P., von Roth, P., Jennings, M. T., Hanssen, A. D., \& Pagnano, M. W. (2016). What Safe Zone? The Vast Majority of Dislocated THAs Are Within the Lewinnek Safe Zone for Acetabular Component Position. Clin Orthop Relat Res, 474(2), 386-391. Retrieved from http://dx.doi.org/10.1007/s11999-015-4432-5. doi:10.1007/s11999-015-4432-5

Charnley, J. (1972). The long-term results of low-friction arthroplasty of the hip performed as a primary intervention. J Bone Joint Surg Br, 54(1), 61-76. Retrieved from http://dx.doi.org/.

Elkins, J. M., Callaghan, J. J., \& Brown, T. D. (2015). The 2014 Frank Stinchfield Award: The 'landing zone' for wear and stability in total hip arthroplasty is smaller than we thought: a computational analysis. Clin Orthop Relat Res, 473(2), 441-452. Retrieved from http://dx.doi.org/10.1007/s11999-014-3818-0. doi:10.1007/s11999-014-3818-0

Lewinnek, G. E., Lewis, J. L., Tarr, R., Compere, C. L., \& Zimmerman, J. R. (1978). Dislocations after total hip-replacement arthroplasties. J Bone Joint Surg Am, 60(2), 217-220. Retrieved from http://dx.doi.org/.

McCollum, D. E., \& Gray, W. J. (1990). Dislocation after total hip arthroplasty. Causes and prevention. Clin Orthop Relat Res(261), 159-170. Retrieved from http://dx.doi.org/.

Reina, N., Putman, S., Desmarchelier, R., Sari Ali, E., Chiron, P., Ollivier, M., . . Fessy, M. H. (2017). Can a target zone safer than Lewinnek's safe zone be defined to prevent instability of total hip arthroplasties? Case-control study of 56 dislocated THA and 93 matched controls. Orthop Traumatol Surg Res, 103(5), 657-661. Retrieved from http://dx.doi.org/10.1016/j.otsr.2017.05.015. doi:10.1016/j.otsr.2017.05.015 